\title{
La parkine est responsable d'un syndrome parkinsonien de transmission autosomique récessive
}

Près de deux siècles après sa description, la physiopathologie de la maladie de Parkinson reste encore largement inconnue. Dans cette affection neurodégénérative, la classique triade bradykinésie, rigidité et tremblement de repos est associée à une bonne réactivité, au moins initiale, à la levodopa. Les lésions neuropathologiques consistent en une perte massive des neurones dopaminergiques de la voie nigro-striatale associée à des corps de Lewy. Ces inclusions éosinophiles, riches en neurofilaments, constituent le stigmate histopathologique de la maladie. Parmi les diverses hypothèses émises, la piste d'une origine génétique a été récemment étayée par l'implication du gène de la synucléine $\alpha$ dans une forme de transmission autosomique dominante $\left(\mathrm{m} / \mathrm{s} 1997, n^{\circ} 10, p .1218\right)$ [1]. Malgré la découverte d'une nouvelle mutation de ce gène dans une famille allemande [2], son séquençage systématique dans plusieurs dizaines de familles avec une transmission autosomique dominante n'a pas permis de mettre en évidence de nouvelles mutations $[3,4]$. Alors qu'il apparaît maintenant que le gène codant pour la synucléine $\alpha$ représente un gène minoritaire en terme de fréquence dans cette maladie, l'attention se porte sur la parkine impliquée dans un syndrome parkinsonien de transmission autosomique récessive $\left(\mathrm{m} / \mathrm{s} 1998, n^{\circ} 8-9\right.$, p. 971$)$ [5].

Les syndromes parkinsoniens de début précoce (avant l'âge de 40 ans) ont été décrits dans de nombreuses populations. Cependant, leur nature souvent familiale n'a été vraiment reconnue qu'au Japon où ils représentent $5 \%$ à $10 \%$ des cas de mala- die de Parkinson. Dans ce pays, de nombreuses familles ont êté décrites sous l'acronyme AR-JP (autosomal recessive juvenile parkinsonism) dont l'analyse a conduit à localiser le gène responsable sur le bras long du chromosome 6 [6]. L'identification de ce nouveau locus appelé PARK2 a permis de tester l'hypothèse d'une liaison dans des familles non japonaises. Des résultats en faveur d'une liaison génétique ont été récemment obtenus dans des familles européennes, d'Afrique du Nord ou du MoyenOrient [7, 8]. Il s'agit probablement d'un locus commun puisque la liaison génétique avec le locus PARK2 est fortement suspectée dans sept des dix familles européennes analysées. Dans une famille japonaise [9] et une famille algérienne avec consanguinité [8], la délétion à l'état homozygote d'un ou plusieurs marqueurs de type microsatellite a contribué à définir la position du gène responsable. Enfin, Kitada et al. [5] ont identifié le gène PARKINE, un nouveau gène codant pour une protéine de 465 acides aminés de fonction inconnue, dont les exons 4 ou $3-7$ sont délétés dans 4 familles japonaises. Il s'agit d'un gène de grande taille (plus de $500 \mathrm{~kb}$ ) qui comporte 12 exons. Une étude européenne [10] met en évidence des délétions différentes dans trois familles (algérienne, française et portugaise). Cependant, la pathologie moléculaire ne s'arrête pas à la délétion d'un ou plusieurs exons puisqu'aucune délétion n'est détectée dans neuf des douze familles consanguines liées au locus PARK2 [10]. Il y a fort à parier que les mutations ponctuelles de ce gène sont fréquentes et que leur caractérisation pourrait contribuer à établir des relations structure-fonction de la protéine parkine.

Ces études montrent que les mutations de la parkine ne sont pas limitées à la population japonaise et que PARKINE pourrait constituer un gène important dans les formes familiales précoces. Elles contribuent également à en définir le phénotype (Tableau I). Les patients PARK2 présentent la triade parkinsonienne classique et répondent de façon excellente au traitement par la levodopa. Cependant, il existe des différences cliniques avec la maladie de Parkinson idiopathique: (1) l'âge de début est plus précoce, mais il ne s'agit pas d'un critère absolu puisqu'on a observé l'apparition de la maladie à 55 ans chez des sujets porteurs d'une délétion de la parkine; (2) le syndrome parkinsonien est parfois associé à une dystonie qui est souvent inaugurale; (3) l'évolution est très peu progressive et la réponse à la levodopa reste très bonne, même après plusieurs decennies, mais les patients sont très gênés par les dyskinésies qui apparaissent précocement au cours du traitement. Il reste à déterminer si la parkine est aussi impliquée dans les cas isolés de début précoce et si ce gène constitue un facteur de susceptibilité pour la maladie de Parkinson idiopathique. Le mode de transmission de la maladie et l'observation de délétions à l'état homozygote démontrent qu'il s'agit d'une affection due à une perte de fonction de la parkine. Il est difficile d'établir un lien entre l'expression de ce gène et la perte sélective des neurones dopaminergiques. Tout d'abord, le transcrit correspondant est exprimé dans la substantia nigra -

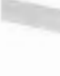

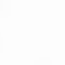

(1)

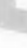

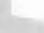

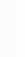

ㄱ․

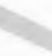

然

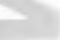

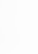

.

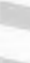

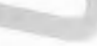


Tableau I

CARACTÉRISTIQUES DES PATIENTS AVEC MUTATION DU GËNE DE LA PARKINE ET AVEC UNE MALADIE DE PARKINSON IDIOPATHIQUE

\begin{tabular}{|lcc|}
\hline & $\begin{array}{c}\text { Mutation } \\
\text { de la parkine }\end{array}$ & $\begin{array}{c}\text { Maladie de Parkinson } \\
\text { idiopathique }\end{array}$ \\
\hline Áge moyen de début (ans) & $\approx 30$ & $\approx 60$ \\
Sex ratio & $1: 1$ & $\mathrm{H}>\mathrm{F}$ \\
Réponse initiale à la levodopa & +++ & ++ \\
Epuisement de la réponse & 0 & ++ \\
Progression de la maladie & lente & +++ \\
Dyskinésies & +++ & ++ \\
Perte neuronale dans & focale & diffuse \\
la substantia nigra & & +++ \\
Corps de Lewy dans le système nerveux & 0 & \\
\hline
\end{tabular}

mais également de façon ubiquitaire dans le système nerveux et dans de nombreux autres tissus [5]. La nature extrêmement sélective des lésions reste à expliquer. La parkine présente une homologie avec les protéines de la famille de l'ubiquitine à son extrémité amino-terminale et comporte un motif RING-finger à son extrémité carboxy-terminale. La piste de l'ubiquitine semble intéressante. En effet, les corps de Lewy dans la maladie de Parkinson comportent de nombreuses protéines ubiquitinées. Une anomalie de la parkine pourrait retentir sur la voie de dégradation liée à l'ubiquitine. Cependant, les deux patients japonais avec AR-JP dont le cerveau a été analysé, présentent une perte massive des neurones de la substantia nigra pars compacta mais pas de corps de Lewy. L'établissement de modèles cellulaires ou ani- maux devrait contribuer à comprendre la fonction de la parkine ainsi que les conséquences de ses anomalies sur les neurones dopaminergiques.

A.B.

\section{Remerciements} français et européens d'étude génétique de la Maladie de Parkinson ainsi que la contribution financière de l'AP-HP, de l'Association France Parkinson et de la Communauté Européenne (Biomed 2).

1. Polymeropoulos $\mathrm{MH}$, Lavedan C, Leroy E, et ah Mutation in the alpha-svnuclein gene identified in families with Parkinson's disease. Science 1997 ; $276: 2045.7$

2. Krůger R, Kuhn W, Muller T, Woitalla D, Graeber M, Kosel S, Przuntek H, Epplen JT, Schols L. Riess $\mathrm{O}$. Ala30Pro mutation in the gene encoding
L'auteur remercie la contribution des réseaux alpha-synuclein in Parkinson's disease. Nat Gemet $1998 ; 18: 106-8$.

3. Vaughan JR, Farrer MJ, W'szolek ZK, el al and the European Consortium on Genetic Susceptibility in Parkinson's Disease (GSPD). Sequencing of the alpha-synuclein gene in a large series of cases of familial Parkinson's disease fails to reveal anv further mutations. Hum Mol Genet $1998 ; 7$ : 751 -3. 4. French Parkinson's Disease Genetics Studv Group. Alpha-synuclein gene and Parkinson's disease. Science $1998 ; 279: 1116-7$.

5. Kitada T, Asakawa S, Hattori N, Matsumine H. Yamamura Y, Minoshima S, Yokochi M, Mizuno Y, Shimizu N. Mutations in the parkin gene cause autosomal recessive juvenile parkinsonism. Nature $1998: 392: 605-8$.

6. Matsumine H, Saito M, Shimoda-Matsubayashi $\mathrm{S}$, et al. Localization of a gene for an autosomal recessive form of juvenile Parkinsonism to chromosome 6q25.2-27. Am J Hum Genet 1997; 60: $588-96$.

7. Jones AC, Yamamura Y, Almasv L, et aL Autosomal recessive juvenile parkinsonism maps to $6 \mathrm{q} 25.2-\mathrm{q} 27$ in four ethnic groups: detailed genetic mapping of the linked region. Am J Hum Genet $1998 ; 63: 80-7$

8. Tassin J, Dürr A, De Broucker T, et al. the French Parkinson's Disease Genetic Study Group and the European lousockum on Genetics Suscepubility in Parkinson's disease. Chromosome 6linked autosomal recessive early-onset Parkinsonism: linkage in European and Algerian families, extension of the clinical spectrum, and evidence of a small homozygous deletion in one family. Am J Hum Genel $1998 ; 63: 88-94$

9. Matsumine H, Yamamura Y, Hattori N, Kobayashi T, Kitada T, Yoritaka A, Mizuno Y. A microdeletion of D6S305 in a family of autosomal recessive juvenile parkinsonism (PARK2). Genomics $1998 ; 49: 143-6$.

10. Lücking $C B$, Abbas N, Dürr A, Bonifati V, Bonnet AM, De Broucker T, De Michele G, Wood NW, Agid Y, Brice A, for the European Consortium on Genetic Susceptibility in Parkinson's Disease and the French Parkinson's Disease Genetics Study Group (1998). Homozygous deletions in the Parkin gene in European and North African families with autosomal recessive juvenile parkinsonism. Lancet 1998; 352: $1355-6$.

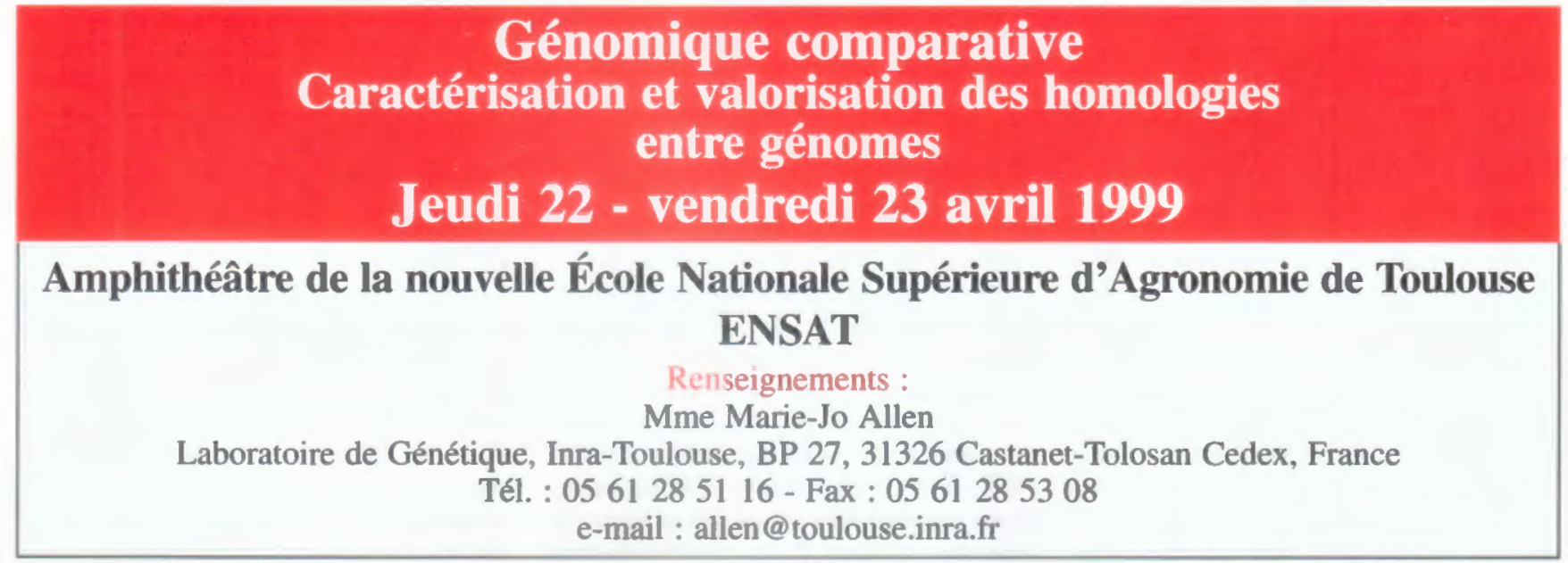

